\title{
Scoliosis and Gastroesophageal Reflux Disease in Adults
}

Fahri Eryilmaz ${ }^{1}$, Faheem Ahmed ${ }^{2}$, Asim K. Rehmani ${ }^{3}$, Sundas Karimi ${ }^{4}$, Aamna Qazi ${ }^{5}$, Sufyan Mustafa ${ }^{6}$, Arif Zulfiqar ${ }^{7}$, Zubia Nadeem ${ }^{5}$, Ayyaz A. Sultan ${ }^{8}$, Umar Farooque ${ }^{9}$

1. Neurological Surgery, Hitit University Corum Erol Olcok Training and Research Hospital, Corum, TUR 2. Orthopedic Surgery, Trauma Centre, Civil Hospital, Karachi, PAK 3. Neurological Surgery, National Medical Center, Karachi, PAK 4. Orthopedic Surgery, Dow University Hospital, Karachi, PAK 5. Medicine, Dow University of Health Sciences, Karachi, PAK 6. Medicine, Dow Medical College, Civil Hospital, Karachi, PAK 7. Medicine and Surgery, Dow Medical College, Karachi, PAK 8. Hematology/Oncology, California Cancer Associates for Research and Excellence, Fresno, USA 9. Neurology, Dow University of Health Sciences, Karachi, PAK

Corresponding author: Umar Farooque, umarfarooque65@gmail.com

\section{Abstract}

\section{Introduction}

Degenerative scoliosis most commonly presents with lower back pain. Literature suggests that adults who have degenerative scoliosis are at greater risk of both hiatal hernia and gastroesophageal reflux disease (GERD). The objective of this study was to evaluate scoliosis as being the risk factor of GERD in adults.

\section{Materials and methods}

This prospective study was conducted at Dow University of Health Sciences over a period of two years (May 2018 to April 2020). The investigation included 210 participants with spinal disorders. The mean age was $71.6 \pm 9.6$ years. The X-rays of the participants' whole spine were taken in a standing position, in the sagittal and coronal planes. Symptoms of GERD were measured through the quality of life and utility evaluation survey technology (QUEST) score, taking six points as cutoff values. The evaluation was done using radiographs to determine any relationship between spinal disorders and GERD. Negative values were analyzed in a right-sided convex curve while positive values in the left-sided convex curve were viewed in the coronal plane. Degenerative scoliosis was explained as a lumbar/thoracolumbar Cobb angle of more than 10 degrees. Univariate and multivariate logistic regression analyses were done to assess the risk factors related to GERD.

\section{Results}

Out of 210 patients, 146 were found to have degenerative scoliosis at the level of the lumbar and thoracolumbar spine. Fifty-two patients had a right convex curve, and 94 had a left convex curve. Sixty-nine patients had GERD. According to the analysis of the multivariate logistic regression, the Cobb angle was highly related to GERD (p-value $<0.05$ and odds ratio of 1.031). The participants were grouped according to the Cobb angle of curve at the lumbar spine (less than 30 degrees with a large right-sided convex curve, 30 and more with a small curve, and more than $30+$ degrees with a large left-sided convex curve). The study revealed that a large left-sided convex curve was highly related to GERD, with a p-value $<0.05$ and odds ratio of 10.935 .

\section{Conclusions}

The left-sided large convex curve at the thoracolumbar or lumbar spine, especially when the Cobb angle was more than 30 degrees, was highly associated with GERD. Therefore, the symptoms of GERD should be monitored in the elderly population with degenerative scoliosis.

Categories: Gastroenterology, Neurosurgery, Orthopedics

Keywords: gastroesophageal reflux disease, scoliosis, quest score, cobb angle, sagittal plane

\section{Introduction}

Lower back pain is a common presentation in patients with degenerative scoliosis. Other symptoms such as leg pain, improper balance in the sagittal and coronal planes, and neurological deficits may appear as well $[1,2]$. It can be elucidated as a Cobb angle of more than 10 degrees in the coronal plane in patients with a mature skeleton [3]. According to a study in the United States, approximately $8.9 \%$ of individuals aged 40 years were found to have lumbar scoliosis, which showed a close relationship between scoliosis and age. The risk of having scoliosis increases from the fifth to the sixth decade of life, with a high prevalence in individuals more than 90 years of age $[4,5]$. Therefore, spinal deformity can be expected in elderly populations. The spinal deformity can cause both musculoskeletal (MSK) and viscera-related disorders, especially in organs near the spine [6,7]. The patients with severe scoliosis at the thoracic region are more 
prone to problems associated with the pulmonary system, such as reduced total lung capacity (TLC) and decreased forced vital capacity (FVC), which are highly associated with idiopathic scoliosis $>40$ degrees and kyphosis $>50$ degrees $[8]$.

The adults who have a degenerative spine with sagittal deformities such as lumbar kyphosis or lumbar and thoracic vertebral fractures were found to be at greater risk of hiatal hernia and gastroesophageal reflux disease (GERD) [9]. Defined as the backflow of gastric and duodenal contents into the esophagus, GERD causes acid regurgitation and heartburn. According to Bari et al., the symptoms of GERD are highly affected by the muscular strength of the back, the lordosis angle of the lumbar, and sagittal balance [10]. The pathophysiology behind GERD determines the impaired functions related to the mechanisms of anti-reflux such as esophageal peristalsis, the lower esophageal sphincter, the pressure gradient of the thoracoabdominal region, and diaphragmatic impairment [10]. Because of the weakened lower esophageal sphincter that allows the retrograde flow of contents into the esophagus, hiatal hernias also cause GERD. The reflux symptoms are prevalent in approximately 10-20\% of the European and North American population and 6.5-9.5\% of the population in Japan. In addition, the risk of having GERD increases with aging $[9,11]$. It is prevalent in approximately $37.6 \%$ of the population of Japan, according to the questionnaire frequency scale for the symptoms of gastroesophageal reflux disease (FSSG) and the quality of life and utility evaluation survey technology (QUEST) [9]. However, the relation between GERD and scoliosis was not analyzed in adults. Therefore, the goal of our study was to assess the effects of truncal deformity on GERD in adults.

\section{Materials And Methods}

This prospective study was conducted at Dow University of Health Sciences for a period of two years, from May 2018 to April 2020. The participants of age 40 years or more and both with and without spinal deformity took part in the study. The patients with spinal deformity underwent a radiographic investigation of the whole spine, in the standing position in both the coronal and the sagittal planes. The data were collected using the QUEST questionnaire. About 246 patients underwent a spinal X-ray and gave QUEST results. Thirty-six participants were not included in the study; 29 were excluded because they gave incomplete data in QUEST, six were excluded due to their history of spinal surgery, and one was excluded because of taking oral steroids. None of the patients revealed any history of previous surgery of esophageal, duodenal, or gastric diseases. Almost 210 participants with a mean age of about $71.6 \pm 9.6$ years and a range of about 45 to 85 years old were included; 61 were male and 149 were female. The diagnoses revealed degenerative lumbar spondylosis, kyphoscoliosis, lumbar stenosis, cervical myopathy, cervical degeneration, and spondylosis. The data regarding height, weight, and drug-related history were evaluated.

QUEST contains seven items to analyze the sensation felt by patients and as an association between the symptoms and exacerbation or relieving factors. The items were allocated according to negative, positive, or neutral scores, which were then added to get a range from seven to +18 . The Japanese QUEST has specificity and sensitivity of $74 \%$ and $65 \%$, respectively, with a $73 \%$ rate of accuracy when six points are set as cut-off values.

According to this study, the six-point QUEST score was considered a positive result of GERD. The radiographic evaluation was done in the lateral view, showing the thoracic vertebra fifth to 12th (T5-T12), the thoracolumbar region twelfth thoracic vertebra to the second lumbar spinal vertebra (T12-L2), and the lumbar lordosis angles at the level of the twelfth thoracic vertebra to sacral spinal nerve one (T12-S1). The pelvic tilt and incidence and the sacral slope and sagittal balance were noted. The lordosis was taken as a negative value and the kyphosis was taken as a positive value. The coronal balance was defined as the alignment of the spine between the $\mathrm{C} 7$ plumb line, and the central vertical line of the sacrum was noted in the frontal view. If the $\mathrm{C} 7$ plumb line was on the right side, the value was taken as positive; if it was on the left side, the value was taken as negative. The Cobb angle of the thoracolumbar and lumbar regions was noted. To differentiate the convex curves, the right-sided was taken as negative and the left-sided was taken as positive. Patients with a Cobb angle of more than 10 degrees were analyzed for degenerative scoliosis. The standard techniques were used to evaluate the radiographic results.

The statistical analysis was done using the Statistical Package for the Social Sciences (SPSS) version 21 (Armonk, NY: IBM Corp.), and the mean \pm standard deviation (SD) of the data was evaluated. The Pearson or Spearman correlation coefficient analysis was carried out to determine the relationship between the QUEST and the radiographic analysis, which included the Cobb angle, trunk balance, and sagittal alignment. Using multivariate and univariate logistic regression analyses, the odds ratio was evaluated with a confidence interval of approximately $95 \%$ to check for the risk factors related to GERD. The p-value of $\leqslant 0.05$ was taken, which was quite significant.

\section{Results}

The mean values and the characteristics of the patients are given in Table 1 . The mean Cobb angle value of $15.4 \pm 16.4$ degrees was observed in all patients in the thoracic region and $24.6 \pm 21.6$ in the lumbar region. Out of 136 patients who had lumbar degenerative scoliosis (curve $>10$ degrees), 52 had a right-sided convex curve with a Cobb angle < 10 degrees, and 94 had a left-sided convex curve with a Cobb angle $>10$ degrees. 


\section{Cureus}

The results revealed no such magnitude difference of the lumbar curve in both groups with curves in a different direction. About 64 participants had a Cobb angle $<10$ degrees, which was absolutely associated with a straight spine. The mean QUEST score in all patients was 4.7 \pm 5.3 . GERD symptoms were found to be in 69 patients (32.8\%), with the cut-off value set at six points.

\begin{tabular}{|c|c|}
\hline Variables & Values \\
\hline Male/female & $61 / 149$ \\
\hline Age (years) & $71.6 \pm 9.6$ \\
\hline Thoracic Cobb angle (absolute value) (deg.) & $15.4 \pm 16.4(0-91.0)$ \\
\hline Lumbar Cobb angle (absolute value) (deg.) & $24.6 \pm 21.6(0-100)$ \\
\hline \multirow[t]{2}{*}{ The mean of the right lumbar convex curve } & 52 cases \\
\hline & (35.1 \pm 18.2 degrees) \\
\hline \multirow[t]{2}{*}{ The mean of the left lumbar convex curve } & 94 patients \\
\hline & (+34.6 \pm 19.5 degrees) \\
\hline Coronal balance $(\mathrm{cm})$ & $0.8 \pm 3.1$ \\
\hline Thoracic kyphosis angle (T5-T12) (deg.) & $24.6 \pm 16.6$ \\
\hline Thoracolumbar kyphosis angle (T10-L2) (deg.) & $17.2 \pm 17.4$ \\
\hline Lumbar lordosis angle (T12-S1) (deg.) & $27.2 \pm 21.1$ \\
\hline Pelvic incidence (deg.) & $52.3 \pm 11.3$ \\
\hline Pelvic tilt (deg.) & $25.4 \pm 12.2$ \\
\hline Sacral slope (deg.) & $27.9 \pm 10.4$ \\
\hline Sagittal balance $(\mathrm{cm})$ & $6.7 \pm 6.3$ \\
\hline QUEST score & $4.7 \pm 5.3(1-15)$ \\
\hline GERD (+) (QUEST score Z6) & 69 patients \\
\hline
\end{tabular}

\section{TABLE 1: Demographic features of patients}

T5-T12: thoracic vertebra fifth to 12th; T12-L2: 12th thoracic vertebra to second lumbar spinal vertebra; T12-S1: 12th thoracic vertebra to sacral spinal nerve one; QUEST: quality of life and utility evaluation survey technology; GERD: gastroesophageal reflux disease

The tested variables along with the QUEST scores are shown in Table 2. The analysis was done using the Pearson correlation coefficient. The QUEST scores were found to be related positively with the Cobb angle of the lumbar region with the correlation coefficient $=0.270$ and the Cobb angle of the thoracic region negatively correlated with the correlation coefficient $=0.158$. The $\mathrm{p}$-value was taken as $<0.05$. The other variables or sagittal plane parameters such as lumbar lordosis, thoracolumbar kyphosis, and sagittal balance were associated with the QUEST score. The Spearman correlation analysis revealed correlations between the variables. It evaluated a strong relation between the Cobb angle of the thoracic region and the lumbar region with a correlation coefficient $=0.858$ and the relation between the sacral slope (SS) and lordosis of the lumbar spine with a correlation coefficient $=0.762$. 


\section{Cureus}

\begin{tabular}{|c|c|c|}
\hline Variables & Correlation coefficient & p-Value \\
\hline Sex & 0.023 & 0.995 \\
\hline Age & 0.115 & 0.156 \\
\hline Lumbar Cobb angle & 0.270 & $<0.06$ \\
\hline Thoracic Cobb angle & 0.158 & $<0.06$ \\
\hline Thoracic kyphosis angle & 0.096 & 0.243 \\
\hline Coronal balance & 0.061 & 0.493 \\
\hline Lumbar lordosis angle & 0.059 & 0.507 \\
\hline Thoracolumbar kyphosis angle & 0.033 & 0.762 \\
\hline Pelvic tilt & 0.013 & 0.977 \\
\hline Pelvic incidence & 0.029 & 0.9 \\
\hline Sagittal balance & 0.06 & 0.59 \\
\hline Sacral slope & 0.024 & 0.858 \\
\hline
\end{tabular}

TABLE 2: Correlations between the QUEST score and variables (Pearson correlation analysis)

QUEST: quality of life and utility evaluation survey technology

The univariate logistic regression analysis was carried out to evaluate the odds ratios of the risk factors associated with GERD (Table 3). The presence of the symptoms of GERD in the participants was considered as a dependent variable. The odds ratio of the coronal lumbar curve was found to be 1.031 . It was 0.993 for the thoracic curve. The p-value was taken as $<0.05$.

\begin{tabular}{|c|c|c|c|}
\hline Variables & OR & $95 \% \mathrm{Cl}$ & p-Value \\
\hline Sex & 1.106 & $0.556-2.211$ & 0.806 \\
\hline Age & 1.001 & 0.973-1.030 & 0.531 \\
\hline Lumbar Cobb angle & $1.031^{\star}$ & $1.020-1.042$ & $<0.05$ \\
\hline Thoracic Cobb angle & $0.993^{\star}$ & 0.978-1.008 & $<0.05$ \\
\hline Thoracic kyphosis (T5-12) & 1.001 & $0.981-1.021$ & 0.376 \\
\hline Coronal balance & 1.042 & $0.937-1.159$ & 0.578 \\
\hline Lumbar lordosis (T12-S1) & 1.019 & $1.003-1.034$ & 0.281 \\
\hline Thoracolumbar kyphosis (T10-L2) & 1.018 & $1.000-1.037$ & 0.403 \\
\hline Pelvic tilt & 1 & 0.983-1.038 & 0.938 \\
\hline Pelvic incidence & 1.012 & $0.983-1.043$ & 0.899 \\
\hline Sagittal balance & 1.039 & 0.982-1.098 & 0.338 \\
\hline Sacral slope & 1.011 & 0.979-1.045 & 0.949 \\
\hline
\end{tabular}

\section{TABLE 3: Univariate logistic regression analysis}

*Significant p-value.

OR: odds ratio; Cl: confidence interval; T5-T12: thoracic vertebra fifth to 12th; T12-S1: 12th thoracic vertebra to sacral spinal nerve one; T10-L2: 10th thoracic vertebra to second lumbar spinal vertebra 


\section{Cureus}

Table 4 shows the multivariate logistic regression analysis. This analysis was done by excluding the thoracic Cobb angle and the SS, as they possess high rates of correlation coefficient with the Cobb angle and lordosis of the lumbar region.

\begin{tabular}{|c|c|c|c|}
\hline Variables & OR & $95 \% \mathrm{Cl}$ & p-Value \\
\hline Lumbar Cobb angle & $1.031^{*}$ & $1.020-1.043$ & $<0.05$ \\
\hline Sex & 1.029 & $0.470-2.268$ & 0.973 \\
\hline Age & 1.001 & $0.969-1.034$ & 0.561 \\
\hline Thoracic kyphosis (T5-12) & 1.007 & $0.980-1.035$ & 0.846 \\
\hline Coronal balance & 0.989 & $0.882-1.109$ & 0.727 \\
\hline Lumbar lordosis (T12-S1) & 1.018 & $1.000-1.036$ & 0.395 \\
\hline Thoracolumbar kyphosis (T10-L2) & 1.018 & 0.996-1.040 & 0.492 \\
\hline Pelvic tilt & 0.996 & $0.966-1.026$ & 0.366 \\
\hline Sagittal balance & 1.035 & $0.957-1.119$ & 0.551 \\
\hline Pelvic incidence & 1.036 & $0.985-1.089$ & 0.342 \\
\hline
\end{tabular}

\section{TABLE 4: Multivariate logistic regression analysis}

*Significant p-value.

OR: odds ratio; Cl: confidence interval; T5-T12: thoracic vertebra fifth to 12th; T12-S1: 12th thoracic vertebra to sacral spinal nerve one; T10-L2: 10 th thoracic vertebra to second lumbar spinal vertebra

The other variables do not show any correlations as shown in Table 5. The patients were categorized in accordance with the Cobb angle of the lumbar curve.

\begin{tabular}{|c|c|c|c|}
\hline Variables & OR & $95 \% \mathrm{Cl}$ & p-Value \\
\hline Lumbar Cobb angle (deg.) < 30 & 1 & & \\
\hline Lumbar Cobb angle (deg.) $\geq 30$ & $10.935^{\star}$ & $3.261-53.799$ & $<0.05$ \\
\hline Age & 1.002 & $1.959-2.026$ & 0.647 \\
\hline Sex & 1.06 & $1.426-3.160$ & 0.931 \\
\hline Coronal balance & 0.988 & $1.871-2.099$ & 0.722 \\
\hline Thoracic kyphosis (T5-12) & 1.004 & $1.967-2.022$ & 0.691 \\
\hline Thoracolumbar kyphosis (T10-L2) & 1.019 & $1.988-2.030$ & 0.427 \\
\hline Lumbar lordosis (T12-S1) & 1.019 & $1.991-2.027$ & 0.333 \\
\hline Pelvic incidence & 1.036 & $1.974-2.080$ & 0.343 \\
\hline Pelvic tilt & 0.998 & $1.957-2.019$ & 0.442 \\
\hline Sagittal balance & 1.031 & $1.946-2.103$ & 0.601 \\
\hline
\end{tabular}

\section{TABLE 5: Multivariate logistic regression analysis for GERD}

*Significant p-value.

OR: odds ratio; Cl: confidence interval; T5-T12: thoracic vertebra fifth to 12th; T10-L2: 10th thoracic vertebra to second lumbar spinal vertebra; T12S1: 12 th thoracic vertebra to sacral spinal nerve one; GERD: gastroesophageal reflux disease 


\section{Discussion}

The risk factors in causing GERD included smoking, hiatal hernia, overweight, alcohol consumption, negative Helicobacter pylori, and aging. Vertebral fractures or malalignments of the spine in the sagittal plane cause kyphosis, which is also associated with hiatal hernia sizes and GERD. The lumbar kyphosis and the vertebral fractures of the lumbar regions are highly related to GERD [12,13]. Although the lordosis of the lumbar spine, the muscular strength of the back, and sagittal balance are associated with GERD symptoms, the studies did not reveal the relation between deformities in the coronal plane and GERD [12].

Hiatal hernias can be associated with scoliosis in both the adolescent and the adult population. According to a study, approximately $6.2 \%$ of adolescents were observed as having hiatal hernia before surgery for idiopathic scoliosis, which was reduced to $2.1 \%$ after the surgery [14]. The analysis of another study indicated that coronal deformity might be associated with GERD because kyphosis is not common among patients with adolescent idiopathic scoliosis [15].

Even though all of the reports revealed that scoliosis might be associated with GERD, the skeletal evaluations noted from the spinal X-rays in standing were incomplete. Moreover, no study showed any significant relationship between the curve of scoliosis and the symptoms of GERD [16]. This study analyzed the data obtained by X-rays of the whole spine of patients in the frontal as well as the sagittal view in standing, which further assisted in the assessment of spinal deformities with accuracy, as compared with the spinal mouse or chest X-rays of the partial spinal view [17].

Although the sagittal plane parameters revealed positive and negative values in kyphosis and lordosis, respectively, the direction of the curve was not taken into consideration while evaluating the magnitude of the curve in scoliosis. Nonetheless, because of the left-sided location of the gastroesophageal junction, the difference between the right and the left-sided curves can be important in the evaluation of the relation between the symptoms of GERD and scoliosis [18]. Therefore, to analyze the direction of the curve when evaluating the risk factors of GERD, we explained the left-sided convex curve of the thoracolumbar or lumbar region as positive and the right-sided as negative.

This study successfully demonstrated that scoliosis of the thoracolumbar or lumbar region is associated with GERD. The odds ratio was 1.031 and the $95 \%$ confidence interval was 1.010-1.033, showing that the convex curve on the left side (positive value) is a significant risk factor of GERD. The Cobb angle categorized the curve with the left-sided curve more strongly associated with GERD (odds ratio $\{\mathrm{OR}\}=10.935$ ). The anatomical reasons revealed diaphragmatic changes that induce GERD due to the left-sided curve [19]. Because the curve apex may induce distortion of the esophageal hiatus, the attachment of the diaphragm on the spine at the thoracolumbar region may lead to hiatal hernia, which further leads to GERD [19].

While evaluating the spinal deformities in patients having hiatal hernia, in their study, Ushirozako et al. observed that $24 \%$ of the patients had scoliosis, and 12 out of 14 presented with the curve apex at the diaphragm. The left convex curve of the thoracolumbar or lumbar region may increase the pressure of the abdomen by abdominal spaces narrowing and pushing organs such as the stomach and the gastroesophageal junction, further causing acid regurgitation or hiatal hernia [20].

The Ishihara et al. study revealed the mechanism in which the reported case with left convex thoracolumbar neuromuscular kyphoscoliosis was analyzed by CT scan and the results showed the deviation of the axis to the left side between the esophagus and the stomach. The study further elaborated on the improvements in the symptoms of GERD after surgical management of scoliosis in that patient [21].

\section{Conclusions}

The left-sided large convex curve at the thoracolumbar or lumbar was highly associated with GERD and the risk of having the disease increased when the Cobb angle was more than 30 degrees. Therefore, it was concluded that symptoms of GERD should be monitored in elderly patients who have degenerative scoliosis.

\section{Additional Information \\ Disclosures}

Human subjects: Consent was obtained or waived by all participants in this study. Dow University of Health Sciences issued approval IRB-1805/DUHS/Approval/2018/2256. Animal subjects: All authors have confirmed that this study did not involve animal subjects or tissue. Conflicts of interest: In compliance with the ICMJE uniform disclosure form, all authors declare the following: Payment/services info: All authors have declared that no financial support was received from any organization for the submitted work. Financial relationships: All authors have declared that they have no financial relationships at present or within the previous three years with any organizations that might have an interest in the submitted work. Other relationships: All authors have declared that there are no other relationships or activities that could appear to have influenced the submitted work. 


\section{References}

1. Ohba T, Ebata S, Oba H, Oda K, Tanaka N, Koyama K, Haro H: Key radiographic parameters that influence the improvement of postoperative gastroesophageal reflux disease in patients treated surgically for adult spinal deformity with a minimum 2-year follow-up. Spine (Phila Pa 1976). 2020, 45:943-949. 10.1097/BRS.0000000000003459

2. Hasegawa T, Ushirozako H, Yamato Y, et al.: Impact of adult spinal deformity corrective surgery in patients with the symptoms of gastroesophageal reflux disease: a 5-year follow-up report. Eur Spine J. 2020, 29:860869. 10.1007/s00586-020-06300-2

3. Hori Y, Matsumura A, Namikawa T, Kato M, Iwamae M, Nakamura H: Comparative study of the spinopelvic alignment in patients with idiopathic lumbar scoliosis between adulthood and adolescence. World Neurosurg. 2021, 149:309-315. 10.1016/j.wneu.2021.02.031

4. Miller F: Cerebral palsy spinal deformity: etiology, natural history, and nonoperative management . Cerebral Palsy. Miller F, Bachrach S, Lennon N, O'Neil ME (ed): Springer, Cham; 2020. 2nd ed.:1711-1721. 10.1007/978-3-319-74558-9 107

5. Arima H, Hasegawa T, Yamato Y, et al.: Factors associated with improved quality of life outcomes in patients undergoing surgery for adult spinal deformity. Spine (Phila Pa 1976). 2021, 46:384-391. 10.1097/BRS.0000000000003908

6. Imagama S, Ando K, Kobayashi K, et al.: Increase in lumbar kyphosis and spinal inclination, declining back muscle strength, and sarcopenia are risk factors for onset of GERD: a 5-year prospective longitudinal cohort study. Eur Spine J. 2019, 28:2619-2628. 10.1007/s00586-019-06139-2

7. Ohba T, Ebata S, Oba H, Koyama K, Yokomichi H, Haro H: Predictors of poor global alignment and proportion score after surgery for adult spinal deformity. Spine (Phila Pa 1976). 2019, 44:1136-1143. 10.1097/BRS.0000000000003086

8. Tanaka N, Ebata S, Oda K, Oba H, Haro H, Ohba T: Predictors and clinical importance of postoperative coronal malalignment after surgery to correct adult spinal deformity. Clin Spine Surg. 2020, 33:337-341. 10.1097/BSD.0000000000000947

9. Ohba T, Oba H, Koyama K, Oda K, Tanaka N, Fujita K, Haro H: Locomotive syndrome: prevalence, surgical outcomes, and physical performance of patients treated to correct adult spinal deformity. [In Press]. J Orthop Sci. 2020, 10.1016/j.jos.2020.06.012

10. Bari TJ, Hallager DW, Hansen LV, Dahl B, Gehrchen M: Mechanical revision following pedicle subtraction osteotomy: a competing risk survival analysis in 171 consecutive adult spinal deformity patients. Spine Deform. 2021, 9:191-205. 10.1007/s43390-020-00195-1

11. Lee NJ, Lenke LG, Cerpa M, et al.: The 90-day reoperations and readmissions in complex adult spinal deformity surgery. Global Spine J. 2020, 10.1177/2192568220953391

12. Bari TJ, Hallager DW, Hansen LV, Dahl B, Gehrchen M: Reducing revision rates following pedicle subtraction osteotomy surgery: a single-center experience of trends over 7 years in patients with adult spinal deformity. Spine Deform. 2021, 9:803-815. 10.1007/s43390-020-00256-5

13. Weigl DM: Scoliosis in non-ambulatory cerebral palsy: challenges and management . Isr Med Assoc J. 2019, 21:752-755.

14. Tandon MS, Dhingra A, Varma V: Management of patient with scoliosis. Problem Based Learning Discussions in Neuroanesthesia and Neurocritical Care. Prabhakar H, Rajan S, Kapoor I, Mahajan C (ed): Springer, Singapore; 2020. 1st ed.:291-330. 10.1007/978-981-15-0458-7_22

15. Nakashima H, Kanemura T, Satake K, et al.: Lateral approach corpectomy and reconstruction after anterior longitudinal ligament release in cases with fixed kyphosis: a technical note and a preliminary case series. J Clin Neurosci. 2020, 78:164-169. 10.1016/j.jocn.2020.04.084

16. Myers LL, Nerminathan A, Fitzgerald DA, Chien J, Middleton A, Waugh MC, Paget SP: Transition to adult care for young people with cerebral palsy. Paediatr Respir Rev. 2020, 33:16-23. 10.1016/j.prrv.2019.12.002

17. Seshadri SM, Rafaat KT, Brzenski A: Anesthetic implications of duchenne muscular dystrophy and the surgical repair of scoliosis. Clinical Anesthesiology II. Benumof J, Manecke G (ed): Springer, Cham; 2019. 1st ed.:167-191. 10.1007/978-3-030-12365-9_9

18. Lee JJ, Oh SH, Jeong YH, et al.: Surgical strategies for cervical deformities associated with neuromuscular disorders. Neurospine. 2020, 17:513-524. 10.14245/ns.2040464.232

19. Marpole R, Blackmore AM, Gibson N, Cooper MS, Langdon K, Wilson AC: Evaluation and management of respiratory illness in children with cerebral palsy. Front Pediatr. 2020, 8:333. 10.3389/fped.2020.00333

20. Ushirozako H, Hasegawa T, Yamato Y, et al.: L5 pedicle subtraction osteotomy maintains good radiological and clinical outcomes in elderly patients with a rigid kyphosis deformity: a more than 2-year follow-up report. Eur Spine J. 2020, 29:3018-3027. 10.1007/s00586-020-06616-z

21. Ishihara Y, Morishita M, Kanzaki K, Toyone T: Age-related progression of degenerative lumbar kyphoscoliosis: a retrospective study. Spine Surg Relat Res. 2020, 4:229-236. 10.22603/ssrr.2019-0113 